\title{
Derived bracket construction and Manin products
}

\author{
K. UCHINO
}

\begin{abstract}
We will extend the classical derived bracket construction to any algebra over a binary quadratic operad. We will show that the derived product construction is a functor given by the Manin white product with the operad of permutation algebras. As an application, we will show that the operad of prePoisson algebras is isomorphic to Manin black product of the Poisson operad with the preLie operad. We will show that differential operators and Rota-Baxter operators are, in a sense, Koszul dual to each other.
\end{abstract}

\section{Introduction.}

Let $(\mathfrak{g},[\cdot, \cdot], d)$ be a differential graded (dg) Lie algebra. We define a new bracket product by $[x, y]_{d}:=-(-1)^{|x|}[d x, y], x, y \in \mathfrak{g}$. Then the new bracket becomes a Leibniz bracket (so-called Loday bracket). This method of constructing a new product is called a derived bracket construction of Koszul-Kosmann-Schwarzbach (cf. Kosmann-Schwarzbach $[7,8]$ ). The derived bracket construction plays important roles in modern analytical mechanics and in Poisson geometry. It is known that several important brackets, e.g., Poisson brackets, Schouten-Nijenhuis brackets, Lie algebroid brackets, Courant brackets and BV-brackets are induced by the derived bracket construction.

The idea of the derived bracket construction arises in several mathematical areas. For instance, given a $\mathrm{dg}$ associative algebra $(A, d)$, the modified products, $x \vdash y:=-(-1)^{|x|}(d x) y$ and $x \dashv y:=x(d y)$, are both associative. The modified products, which are called the derived products, are used in the study of Loday type algebras (cf. Aguiar [1], Loday [10]).

Mathematics Subject Classifications (2000):18D50, 53D17.

Keywords: derived brackets, operads, perm-algebras, Manin products, Rota-Baxter operators, Poisson brackets. 
The purpose of this letter is to extend the classical derived bracket/product construction to any $\mathcal{P}$ (binary quadratic operad)-algebra . It will be shown that the derived bracket construction is a representation of the functor:

$$
\mathcal{P e r m} \circ(-): \mathcal{P} \mapsto \mathcal{P} \text { erm } \circ \mathcal{P}
$$

where Perm is the operad governing permutation algebras (cf. Chapoton [3]) and where $\circ$ is the white product of Manin-Ginzburg-Kapranov (so-called Main white product). Let $\mathcal{P}$ be a binary quadratic operad and let $A$ be a $\operatorname{dg} \mathcal{P}$-algebra. We regard the differential as a 1-ary operation. We define the derived brackets on $s A$ by the usual manner:

$$
\begin{aligned}
& {[s x, s y]_{d}:=-(-1)^{|x|} s[d x, y]} \\
& {[s x, s y]^{d}:=s[x, d y]}
\end{aligned}
$$

where $s$ is a degree shifting operator and the bracket is a multiplication of $\mathcal{P}$-algebra. The derived brackets generate a new algebra structure on $s A$. In Theorem 3.2, we will show that the algebra generated by the derived brackets is a $\mathcal{P} e r m \circ \mathcal{P}$-algebra. To prove the theorem we apply the detailed study for Manin products (Vallette $[12])$. Vallette showed that the operad of Leibniz algebras, $\mathcal{L} e i b$, is isomorphic to $\mathcal{P}$ erm $\circ \mathcal{L}$ ie (Theorem 20 in [12]). We will show that the theorem of Vallette is the classical derived bracket construction on the level of operad. As an application, it will be shown that the operad of prePoisson algebras ([1]), prePoiss, is isomorphic to the operad pre $\mathcal{L}$ ie $\bullet \mathcal{P}$ oiss, where pre $\mathcal{L}$ ie is the operad of preLie algebras, $\mathcal{P}$ oiss is the operad of Poisson algebras and $\bullet$ is the Manin black product which is the Koszul dual of the white product.

We will discuss a Koszul duality for the derived bracket construction. The differential operators satisfy the relation below.

$$
d[d x, y]=-[d x, d y]=-d[x, d y]
$$

This relation is the heart of the derived bracket construction. We consider the following operator identity on $\mathcal{P}^{\text {! }}$-algebras instead of the derivation relation.

$$
\beta(x) * \beta(y)=\beta(\beta(x) * y+x * \beta(y)),
$$

where $*$ is a multiplication of $\mathcal{P}^{\text {! }}$-algebra. This $\beta$ is called a Rota-Baxter operator. It is known that some integral operators are Rota-Baxter operators. We introduce a new concept, the dual-derived products, which are multiplications defined by the Rota-Baxter operators:

$$
\beta(x) * y \text { and } x * \beta(y) .
$$


The original idea of the dual-derived products was given by Aguiar in [1]. We will prove that the algebras generated by the dual-derived products are pre $\mathcal{L} i e \bullet \mathcal{P}^{\text {! }}$ algebras (Theorem 4.2 below). Since pre $\mathcal{L} i e \bullet \mathcal{P}^{!}$is the Koszul dual of $\mathcal{P} e r m \circ \mathcal{P}$, the dual-derived product construction is a kind of Koszul dual of the derived bracket construction. Therefore, one can regard Rota-Baxter/integral operators as Koszul dual operators of differential operators.

Acknowledgement. The author would like to thank very much referees for kind advice and useful comments and also thank Professor Akira Yoshioka for kind advice.

\section{Preliminaries}

Assumptions. The characteristic of the ground field $\mathbb{K}$ is zero. We follow the standard Koszul sign convention.

We recall the notion of (graded) operad. For details, see Ginzburg-Kapranov $[5,6]$ or Markl-Shnider-Stasheff [11]. In Appendix, we give a short survey of Koszul duality theory and Manin products.

Example 2.1. (endomorphism operad) Let $V$ be a (graded) vector space. We consider a collection of the spaces of linear endomorphisms, $\mathcal{E} n d(V):=\{\mathcal{E} n d(V)(n)\}_{n \in \mathbb{N}}$, $\mathcal{E} n d(V)(n):=\operatorname{Hom}\left(V^{\otimes n}, V\right)$. For any $f \in \mathcal{E} n d(V)(m)$ and $g \in \mathcal{E} n d(V)(n)$, we define an ith-composition by

$f \circ{ }_{i} g\left(x_{1}, \ldots, x_{m+n-1}\right):=(-1)^{|g|\left(\left|x_{1}\right|+\cdots+\left|x_{i-1}\right|\right)} f\left(x_{1}, \ldots, x_{i-1}, g\left(x_{i}, \ldots, x_{i+n-1}\right), x_{i+n}, \ldots, x_{m+n-1}\right)$. where $|\cdot|$ is the degree of the object. The composition is a binary map:

$$
\circ_{i}: \mathcal{E} n d(V)(m) \otimes \mathcal{E} n d(V)(n) \rightarrow \mathcal{E} n d(V)(m+n-1) .
$$

The composition is associative in the sense of (1) below.

$$
\left(f \circ \circ_{i} g\right) \circ_{j} h= \begin{cases}(-1)^{|g||h|}\left(f \circ_{j} h\right) \circ_{i+l-1} g & 1 \leq j \leq i-1 \\ f \circ_{i}\left(g \circ_{j-i+1} h\right) & i \leq j \leq i+n-1 \\ (-1)^{|g||h|}\left(f \circ_{j-n+1} h\right) \circ_{i} g & i+n \leq j \leq m+n-1,\end{cases}
$$

where $f \in \mathcal{E} n d(V)(m), g \in \mathcal{E} n d(V)(n)$ and $h \in \mathcal{E} n d(V)(l)$. The space $\mathcal{E} n d(V)(n)$ has a canonical $S_{n}$-module structure defined by

$$
f \sigma\left(x_{1}, \ldots, x_{n}\right):=\epsilon(\sigma) f\left(x_{\sigma^{-1}(1)}, \ldots, x_{\sigma^{-1}(n)}\right),
$$

where $\sigma \in S_{n}$ and $\epsilon(\sigma)$ is Koszul sign. The composition is equivariant, that is,

$$
f \sigma \circ_{i} g \tau=\left(f \circ_{\sigma^{-1}(i)} g\right)\left(\sigma \circ_{i} \tau\right)
$$


where $\sigma \circ_{i} \tau \in S_{m+n-1}$. There exists the identity map id $: V \rightarrow V$ in $\mathcal{E} n d(V)(1)$. The identity map is the unit element with respect to the composition:

$$
f \circ_{i} i d=f=i d \circ_{1} f .
$$

Let $E_{n}$ be a (graded) vector space and an $S_{n}$-module. A collection of such spaces, $\left\{E_{n}\right\}_{n \in \mathbb{N}}$, is called an $S$-module.

Definition 2.2. An operad is an $S$-module, $\{\mathcal{P}(n)\}_{n \in \mathbb{N}}$, equipped with a collection of compositions, $\left\{o_{i}: \mathcal{P}(m) \otimes \mathcal{P}(n) \rightarrow \mathcal{P}(m+n-1)\right\}$, and the unit element $1 \in \mathcal{P}(1)$ satisfying (1), (2) and (3).

Definition 2.3. An operad morphism, $\phi: \mathcal{P}_{1} \rightarrow \mathcal{P}_{2}$, is a collection of $S_{n}$-equivariant linear maps of degree $0,\left\{\phi(n): \mathcal{P}_{1}(n) \rightarrow \mathcal{P}_{2}(n)\right\}_{n \in \mathbb{N}}$, which commute the operad composition maps and which preserves the unit.

Example 2.4. (cf. [5] Example 2.1.10) The operad of Lie algebras, $\mathcal{L} i e$, is an $S$ module $\{\mathcal{L} i e(n)\}_{n \in \mathbb{N}}$ which is generated by a 1-dimensional vector space $\mathcal{L} i e(2):=$ $\langle\mu\rangle$. We assume $\mathcal{L} i e(1):=\mathbb{K}$. The $S_{2}$-action on $\mathcal{L} i e(2)$ is given by a sign representation (i.e. skewsymmetry). The generating relation of the Lie operad is the Jacobi identity,

$$
\mu \circ_{2} \mu-\mu \circ_{1} \mu-\left(\mu \circ_{2} \mu\right)((12) \otimes 1), \quad(12) \in S_{2},
$$

or explicitly, $\mu\left(x_{1}, \mu\left(x_{2}, x_{3}\right)\right)-\mu\left(\mu\left(x_{1}, x_{2}\right), x_{3}\right)-\mu\left(x_{2}, \mu\left(x_{1}, x_{3}\right)\right)$.

If $(\mathfrak{g},[\cdot, \cdot])$ be a Lie algebra, then there exists a suboperad of the endomorphism operad $\mathcal{E} n d(\mathfrak{g})$ which is generated by the Lie bracket $[\cdot, \cdot]: \mathfrak{g} \otimes \mathfrak{g} \rightarrow \mathfrak{g}$. This suboperad is a representation of the operad $\mathcal{L} i e$. Conversely, given an operad morphism (representation) rep $: \mathcal{L} i e \rightarrow \mathcal{E} n d(V), V$ becomes a Lie algebra.

Definition 2.5. Let $\mathcal{P}$ be an operad. The $\mathcal{P}$-algebra structure is defined to be an operad representation,

$$
r e p: \mathcal{P} \rightarrow \mathcal{E} n d(V)
$$

The notion of $\mathcal{P}$-algebra is defined by this way.

Example 2.6. (Leibniz algebras; Leib; cf. [9, 10]) A (left) Leibniz algebra (also called, a Loday algebra) is a (graded) vector space with a binary multiplication satisfying the (left) Leibniz identity,

$$
[x,[y, z]]=[[x, y], z]+(-1)^{|x||y|}[y,[x, z]] .
$$


When the bracket is skewsymmetric, it is a Lie algebra. The operad of Leibniz algebras, $\mathcal{L}$ eib, is generated by a 2-dimensional vector space $\left\langle\mu, \mu^{(12)}\right\rangle$, where $\mu^{(12)}$ is the transposition of $\mu$, that is,

$$
\mu^{(12)}\left(x_{1}, x_{2}\right)=\mu\left(x_{2}, x_{1}\right)
$$

The generating relation of $\mathcal{L}$ eib is the same as the relation of $\mathcal{L} i e$.

Example 2.7. (Permutation-algebras; Perm; cf. [3, 4], [12] Section 4.1) A (graded) vector space with a binary multiplication $(C, *)$ is called a (left) permutation algebra (or called a perm-algebra for short), if $*$ is associative and satisfies (4) below.

$$
(x * y) * z-(-1)^{|x||y|}(y * x) * z=0,
$$

where $x, y, z \in C$. One can check that the nth-component of the operad of permalgebras is isomorphic to $\mathbb{K}^{n}$ for each $n \in \mathbb{N}$. Therefore the dimension relation below holds.

$$
\operatorname{dim} \operatorname{Perm}(n)=n, \quad \text { for each } n \text {. }
$$

We will use this relation in the next section. We denote the linear basis of $\mathcal{P} \operatorname{erm}(n)$ by $\left\langle e_{1}^{n}, \ldots, e_{n}^{n}\right\rangle$. Here $e_{j}^{n}$ corresponds with an $n$-monomial whose right component is $x_{j}$ :

$$
e_{j}^{n} \cong\left(x_{i_{1}} * \cdots * x_{i_{n-1}}\right) * x_{j},
$$

where $i_{a} \in\{1, \ldots, \check{j}, \ldots, n\}$. It is known that the composition rule of Perm is given by

$$
e_{i}^{m} \circ_{j} e_{k}^{n}=\left\{\begin{array}{cc}
e_{i}^{m+n-1} & i<j \\
e_{i+k-1}^{m+n-1} & j=i \\
e_{i+n-1}^{m+n-1} & i>j
\end{array}\right.
$$

We will use these relations in the next section.

Example 2.8. Let $\mathcal{P}_{1}, \mathcal{P}_{2}$ be operads. Then $\mathcal{P}_{1} \otimes \mathcal{P}_{2}:=\left\{\mathcal{P}_{1}(n) \otimes \mathcal{P}_{2}(n)\right\}_{n \in \mathbb{N}}$ becomes an operad by a natural manner.

We recall the free operad.

Example 2.9. (cf. [5] Section 2.1.1, [11] page 71) Let $E:=\left(E_{1}, E_{2}, \ldots\right)$ be an $S$ module. We identify an element $f$ in $E_{n}$ with the $n$-corolla (a tree with one vertex and $n$-leaves) whose vertex is decorated with $f$. For instance, $f \in E_{3}$,

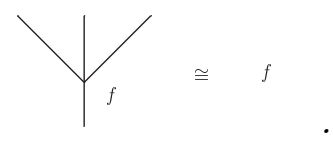


Given $f \in E(m), g \in E(n)$, grafting g-corolla on the ith-leaf of $f$-corolla, one can define an operad composition $f \circ_{i} g$, namely, $f \circ_{i} g$ is a tree whose vertices are decorated with $f$ and $g$. For instance, if $f \in E_{3}, g, h \in E_{2}$, then $\left(g \circ_{1} f\right) \circ_{4} h=$

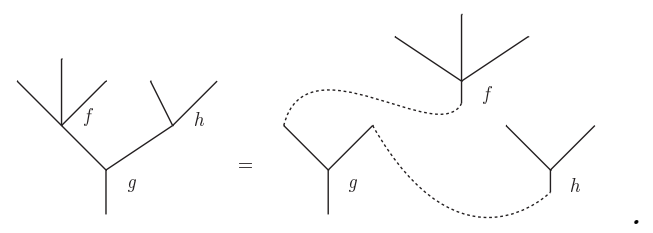

In this way, an operad, which is denoted by $\mathcal{F}(E)$, is generated. This is the free operad over the $S$-module. Incidentally, the example $\left(g \circ_{1} f\right) \circ_{4} h$ is an element in $\mathcal{F}(E)(5)$.

Let $E$ be a (graded) vector space. We identify $E$ with an $S$-module such that $\left\{E_{2}=E, E_{n \neq 2}=0\right\}$. We consider the free operad $\mathcal{F}(E)$. A subspace $R \subset \mathcal{F}(E)(3)$ is called a quadratic relation, if it is $S_{3}$-stable. Let $R$ be a quadratic relation and let $(R)$ be the generated operad ideal of the free operad. The quotient operad $\mathcal{P}(E, R):=\mathcal{F}(E) /(R)$ is called a binary quadratic operad. The above operads $\mathcal{L} i e$, Leib and $\mathcal{P} e r m$ are binary quadratic operads. One can show that $\mathcal{F}(E)(3)$ is generated by the elements of the form:

$$
e \circ_{1} e^{\prime}\left(x_{i}, x_{j}, x_{k}\right)
$$

where $e, e^{\prime} \in E$ and $(i, j, k) \in\{(1,2,3),(3,1,2),(2,3,1)\}$. We identify $e o_{1} e^{\prime} \cong e \otimes e^{\prime}$. Then we obtain the following isomorphisms.

$$
\mathcal{F}(E)(3) \cong \bigoplus_{(i, j, k)} E \otimes E \otimes(i, j, k) \cong 3 E \otimes E
$$

This gives the dimension relation for the binary quadratic operad:

$$
\operatorname{dim} \mathcal{P}(3)=3(\operatorname{dim} E)^{2}-\operatorname{dim} R
$$

We will use this relation in the next section.

We recall the shifted operads.

Example 2.10. (cf. [5] Definition 3.2.13; [11] page 127) The shifted operad of $\mathcal{E} n d(V)$ (which is denoted by $\mathbf{s} \mathcal{E} n d(V)$ ) is, by definition, $\mathcal{E} n d(s V)$, where $s$ is the degree shifting operator of degree $|s|=+1$. An arbitrary element $\mathbf{s} f \in \mathbf{s} \mathcal{E} n d(V)(n)$ has the form, $\mathbf{s} f=s f\left(s^{-1} \otimes \ldots \otimes s^{-1}\right)$. One can regard $s^{-1} \otimes \ldots \otimes s^{-1}$ as a one dimensional sign representation of $S_{n}$. Thus we obtain

$$
s f\left(s^{-1} \otimes \ldots \otimes s^{-1}\right) \cong f[1-n] \otimes \operatorname{sgn}(n),
$$


where $f[1-n]$ is a copy of $f$ with degree $|f|+1-n$ and $\operatorname{sgn}(n)$ is the one dimensional representation space.

Given an operad $\mathcal{P}$, the shifted operad $\mathbf{s} \mathcal{P}$ is defined by $\mathbf{s} \mathcal{P}:=\mathcal{P}[1-\cdot] \otimes \operatorname{sgn}$ in general, where $\operatorname{sgn}=\{\operatorname{sgn}(n)\}_{n \in \mathbb{N}}$.

\section{Main results}

\subsection{Derived brackets}

Let $\mathcal{P}=\mathcal{P}(E, R)$ be a binary quadratic operad and let $(A, d)$ be a $\operatorname{dg} \mathcal{P}$-algebra. We assume that $E$ is a homogeneous space of degree zero (or even) and assume that $|d|=+1$ (or odd).

Definition 3.1. The derived brackets on $s A$ are defined to be the following binary multiplications,

$$
\begin{aligned}
& {\left[s x_{1}, s x_{2}\right]_{d}:=-(-1)^{\left|x_{1}\right|} s\left[d x_{1}, x_{2}\right]} \\
& {\left[s x_{1}, s x_{2}\right]^{d}:=s\left[x_{1}, d x_{2}\right]}
\end{aligned}
$$

where $[\cdot, \cdot]$ is a $\mathcal{P}$-algebra multiplication and $s x_{1}, s x_{2} \in s A, x_{1}, x_{2} \in A$.

The derived brackets are given by

$$
\begin{aligned}
& {[\cdot, \cdot]_{d}:=s[\cdot, \cdot](d \otimes 1)\left(s^{-1} \otimes s^{-1}\right),} \\
& {[\cdot, \cdot]^{d}:=-s[\cdot, \cdot](1 \otimes d)\left(s^{-1} \otimes s^{-1}\right),}
\end{aligned}
$$

on the level of the endomorphism operad. If $[\cdot, \cdot]$ is (anti-)commutative, then $[\cdot, \cdot]^{d}$ is the (anti-)transposition of $[\cdot, \cdot]_{d}$, namely,

$$
\left[s x_{1}, s x_{2}\right]^{d}=( \pm 1)(-1)^{\left(\left|x_{1}\right|+1\right)\left(\left|x_{2}\right|+1\right)}\left[s x_{2}, s x_{1}\right]_{d} .
$$

Let $\mu$ be an $n$-monomial of $\mathcal{P}$-algebra. It satisfies the derivation property,

$$
d \mu\left(x_{1}, \ldots, x_{n}\right)=\sum( \pm) \mu\left(x_{1}, \ldots, x_{i-1}, d x_{i}, x_{i+1}, \ldots, x_{n}\right),
$$

where \pm is an appropriate sign. Hence the monomials composed of the (nonshifted) derived brackets are generated by the monomials of the form,

$$
\mu \circ \mathbf{d}_{i}^{n}:=\mu\left(d \otimes \ldots \otimes d \otimes 1_{(i t h)} \otimes d \otimes \ldots \otimes d\right) .
$$

For instance, $d[\cdot,[d[\cdot, \cdot], d(\cdot)]]=[d(\cdot),[[d(\cdot), \cdot], d(\cdot)]]+[d(\cdot),[[\cdot, d(\cdot)], d(\cdot)]]=$

$$
=[\cdot[[\cdot, \cdot], \cdot]](d \otimes d \otimes 1 \otimes d)+[\cdot,[[\cdot, \cdot], \cdot]](d \otimes 1 \otimes d \otimes d) .
$$

Our main result is the following. 
Theorem 3.2. The algebra of the derived brackets is a Perm $\circ \mathcal{P}$-algebra.

Vallette showed $\mathcal{P} e r m \otimes \mathcal{P} \cong \mathcal{P} e r m \circ \mathcal{P}$ in [12] Proposition 15 (See Appendix for the definition of Manin products). So it suffices to show that $s A$ becomes a $\mathcal{P}$ erm $\otimes \mathcal{P}$-algebra.

Proof. The monomials composed of the (shifted) derived brackets are generated by the monomials of the form,

$$
\mathbf{s}\left(\mu \circ \mathbf{d}_{i}^{n}\right)=s \mu(d \otimes \ldots \otimes d \otimes 1 \otimes d \otimes \ldots \otimes d)\left(s^{-1} \otimes \ldots \otimes s^{-1}\right) .
$$

We remark that the degree of $\mathbf{s}\left(t \circ \mathbf{d}_{i}^{n}\right)$ is 0 . We have to mix $d$ with $s^{-1}$, that is,

$$
\pm \mathbf{s}\left(\mu \circ \mathbf{d}_{i}^{n}\right)=s \mu\left(d s^{-1} \otimes \ldots \otimes d s^{-1} \otimes s^{-1} \otimes d s^{-1} \ldots \otimes d s^{-1}\right),
$$

where $\pm=(-1)^{n(n-1) / 2+(i-1)}$. We define maps,

$$
\mathcal{P} e r m \otimes \mathcal{P} \stackrel{1 \otimes r e p}{\longrightarrow} \mathcal{P} e r m \otimes \mathcal{E} n d(A) \stackrel{\phi}{\longrightarrow} \mathbf{s} \mathcal{E} n d(A),
$$

where $\phi$ is the collection of $\phi(n)$,

$$
\phi(n): e_{i}^{n} \otimes \mu \mapsto \pm \mathbf{s}\left(\mu \circ \mathbf{d}_{i}^{n}\right)
$$

The map $\phi$ preserves the composition relations of Perm in Example 2.7, namely, the following relations hold.

$$
\pm \mathbf{s}\left(\mu \circ \mathbf{d}_{i}^{m}\right) \circ_{j} \pm \mathbf{s}\left(\mu^{\prime} \circ \mathbf{d}_{k}^{n}\right)=\left\{\begin{array}{cc} 
\pm \mathbf{s}\left(\mu \circ_{j} \mu^{\prime} \circ \mathbf{d}_{i}^{m+n-1}\right) & i<j \\
\pm \mathbf{s}\left(\mu \circ_{j} \mu^{\prime} \circ \mathbf{d}_{i+k-1}^{m+n-1}\right) & i=j \\
\pm \mathbf{s}\left(\mu \circ_{j} \mu^{\prime} \circ \mathbf{d}_{i+n-1}^{m+n-1}\right) & i>j .
\end{array}\right.
$$

This implies that $\phi$ is an operad morphism. Therefore $\phi \circ(1 \otimes r e p)$ is an operad representation. The proof of the theorem is completed.

The proof above shows that the composition of the operad Perm corresponds to the derivation property. However the converse of this correspondence is not clear. In the next subsection we will show that the quadratic relations of $\mathcal{P}$ erm $\circ \mathcal{P}$ are completely determined by the properties that the derived brackets satisfy.

We consider the derived brackets on abelian subalgebras. Let $\mathfrak{g}$ be a dg-Lie algebra. If $\mathfrak{g}^{\prime} \subset \mathfrak{g}$ is an abelian (trivial) subalgebra of $\mathfrak{g}$ (not dg subalgebra) and if $s \mathfrak{g}^{\prime}$ is closed under the derived bracket, then the derived bracket is Lie on $s \mathfrak{g}^{\prime}$. This proposition is one of the basic propositions of the classical derived bracket construction (see [7]). We should state an operadic version of this proposition. 
Proposition 3.3. Let $A$ be a $d g \mathcal{P}$-algebra with a trivial subalgebra $A^{\prime}$ (not $d g$ subalgebra). If $s A^{\prime}$ is closed under the derived brackets, then $s A^{\prime}$ is a $\mathcal{P}$-algebra.

Proof. The commutative associative algebras are obviously permutation-algebras. Hence there exists an operadic projection $p r: \mathcal{P e r m} \rightarrow \mathcal{C}$ om, where $\mathcal{C}$ om is the operad of commutative associative algebras. Since $A^{\prime}$ is a trivial subalgebra, the derived brackets satisfy the following relation on $s A^{\prime}$.

$$
s[\cdot, \cdot]\left(d s^{-1} \otimes s^{-1}\right)=s[\cdot, \cdot]\left(s^{-1} \otimes d s^{-1}\right) .
$$

This implies that the representation of $\mathcal{P}$ erm $\otimes \mathcal{P}$ on $s A^{\prime}$ factors through $\mathcal{C}$ om $\otimes \mathcal{P}$, namely, the following diagram is commutative.

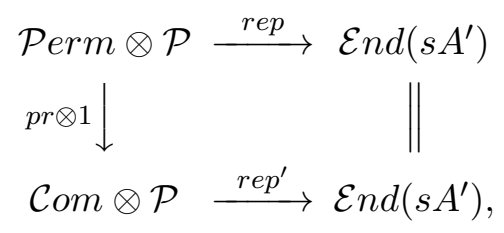

where $r e p^{\prime}$ is the reduced representation. It is known that $\operatorname{Com}(n) \cong \mathbb{K}$ for each $n \in \mathbb{N}$. This gives $\mathcal{C}$ om $\otimes \mathcal{P} \cong \mathcal{P}$. Therefore $s A^{\prime}$ becomes a $\mathcal{P}$-algebra.

\subsection{Free derived bracket}

We reconstruct $\mathcal{P}$ erm $\circ \mathcal{P}$ in terms of the derived bracket construction. First, we give a corollary of Theorem 3.2 .

Corollary 3.4. (Aguiar's derived brackets; cf. [1]) Let $(A, \alpha)$ be a $\mathcal{P}$-algebra with a linear endomorphism $\alpha: A \rightarrow A$. Assume that $\alpha$ satisfies Rota's averaging relation:

$$
\alpha\left[\alpha x_{1}, x_{2}\right]=\left[\alpha x_{1}, \alpha x_{2}\right]=\alpha\left[x_{1}, \alpha x_{2}\right]
$$

Aguiar defined the following modified brackets (derived brackets),

$$
\left[\alpha x_{1}, x_{2}\right] \text { and }\left[x_{1}, \alpha x_{2}\right]
$$

The algebra of Aguiar's derived brackets is a $\mathcal{P}$ erm $\circ \mathcal{P}$-algebra.

Proof. The averaging relation corresponds to the differential relation: $d\left[d x_{1}, x_{2}\right]=$ $-\left[d x_{1}, d x_{2}\right]=-d\left[x_{1}, d x_{2}\right]$, up to shift. Therefore the corollary is shown in a similar manner.

Let $\mathcal{P}$ be a binary quadratic operad over $(E, R)$. Since $\operatorname{dim} \operatorname{Perm}(2)=2$, $\mathcal{P} \operatorname{erm}(2) \otimes \mathcal{P}(2)$ is 2 -copies of $E$. We put

$$
\mathcal{P} \operatorname{Prm}(2) \otimes \mathcal{P}(2):=\left\langle\left[\alpha x_{1}, x_{2}\right],\left[x_{1}, \alpha x_{2}\right] \mid\left[x_{1}, x_{2}\right] \in E\right\rangle,
$$


where $\alpha$ (left and right) is a label on copies (not averaging operator). We define an $S_{2}$-module structure on $\mathcal{P} \operatorname{erm}(2) \otimes \mathcal{P}(2)$ by the natural manner:

$$
\left[\alpha x_{1}, x_{2}\right](12)=\left[\alpha x_{2}, x_{1}\right]=\left[x_{1}, \alpha x_{2}\right]^{(12)},
$$

where $(12) \in S_{2}$ and $[\cdot, \cdot]^{(12)} \in E$ is the transposition of $[\cdot, \cdot]$. One can regard $\left[\alpha x_{1}, x_{2}\right]$ and $\left[x_{1}, \alpha x_{2}\right]$ as binary corollas whose leaves are decorated with symbol $\alpha$ and whose vertices are decorated with $\left[x_{1}, x_{2}\right]$ :

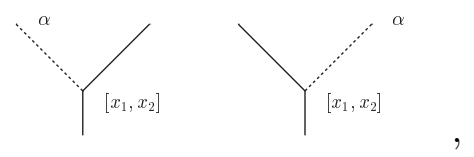

where $\alpha$-edges are drew as broken lines. We consider the free operad $\mathcal{F}(\mathcal{P e r m}(2) \otimes$ $\mathcal{P}(2))$ and the quadratic relation below.

$$
\left[\alpha\left[\alpha x_{i}, x_{j}\right], x_{k}\right]^{\prime}-\left[\alpha\left[x_{i}, \alpha x_{j}\right], x_{k}\right]^{\prime} \equiv 0,
$$

where $[\cdot, \cdot],[\cdot, \cdot]^{\prime} \in E$ and $(i, j, k) \in\{(1,2,3),(3,1,2),(2,3,1)\}$. Let Ave denote the space of relations generated by $(7)$. We set the 3 -copies of $\mathcal{F}(E)(3)$,

$$
3 \mathcal{F}(E)(3):=\left(\mathcal{F}(E)(3) \overline{\mathrm{o}}_{2} \alpha \overline{\mathrm{o}}_{3} \alpha\right) \oplus\left(\mathcal{F}(E)(3) \overline{\mathrm{o}}_{3} \alpha \overline{\mathrm{o}}_{1} \alpha\right) \oplus\left(\mathcal{F}(E)(3) \overline{\mathrm{o}}_{1} \alpha \overline{\mathrm{o}}_{2} \alpha\right),
$$

where $\bar{o}_{i} \alpha \bar{o}_{j} \alpha$ are labels on copies. We define a natural $S_{3}$-module structure on $3 \mathcal{F}(E)$ such that a map $\theta$ below is equivariant. The map $\theta$ is defined to be a surjection $\theta: \mathcal{F}(\mathcal{P e r m}(2) \otimes \mathcal{P}(2))(3) \rightarrow 3 \mathcal{F}(E)(3)$ by

$$
\begin{aligned}
{\left[\alpha\left[\alpha x_{i}, x_{j}\right], x_{k}\right]^{\prime} } & \mapsto\left[\left[x_{i}, x_{j}\right], x_{k}\right]^{\prime} \bar{\circ}_{i} \alpha \overline{\mathrm{o}}_{j} \alpha, \\
{\left[\alpha\left[x_{i}, \alpha x_{j}\right], x_{k}\right]^{\prime} } & \mapsto\left[\left[x_{i}, x_{j}\right], x_{k}\right]^{\prime} \overline{\mathrm{o}}_{i} \alpha \overline{\mathrm{o}}_{j} \alpha, \\
{\left[\left[\alpha x_{i}, x_{j}\right], \alpha x_{k}\right]^{\prime} } & \mapsto\left[\left[x_{i}, x_{j}\right], x_{k}\right]^{\prime} \overline{\mathrm{o}}_{k} \alpha \overline{\mathrm{o}}_{i} \alpha, \\
{\left[\left[x_{i}, \alpha x_{j}\right], \alpha x_{k}\right]^{\prime} } & \mapsto\left[\left[x_{i}, x_{j}\right], x_{k}\right]^{\prime} \overline{\mathrm{o}}_{j} \alpha \overline{\mathrm{o}}_{k} \alpha,
\end{aligned}
$$

where $(i, j, k) \in\{(1,2,3),(3,1,2),(2,3,1)\}$. The relation Ave is the kernel of $\theta$.

Lemma 3.5. The dimension of $\theta^{-1}(3 R)$ is equal to $3(\operatorname{dim} E)^{2}+3 \operatorname{dim} R$, and it coincides with the dimension of the space of quadratic relations of $\mathcal{P}$ erm $\circ \mathcal{P}$.

Proof. One can easily check that $\operatorname{dim} A v e=3(\operatorname{dim} E)^{2}$. This gives the dimension relation $\operatorname{dim} \theta^{-1}(3 R)=3(\operatorname{dim} E)^{2}+3 \operatorname{dim} R$. Since $\mathcal{P e r m} \circ \mathcal{P} \cong \mathcal{P e r m} \otimes \mathcal{P}$ and $\operatorname{dim} \operatorname{Perm}(3)=3$, from (5), we obtain

$$
\operatorname{dim} R_{\mathcal{P} e r m \circ \mathcal{P}}=3(\operatorname{dim} E)^{2}+3 \operatorname{dim} R,
$$

where $R_{\mathcal{P} e r m \circ \mathcal{P}}$ is the relation of $\mathcal{P}$ erm $\circ \mathcal{P}$. 
Since $\theta$ is equivariant, $\theta^{-1}(3 R)$ is a quadratic relation. The main result of this subsection is as follows.

Proposition 3.6. $\mathcal{F}(\mathcal{P} e r m(2) \otimes \mathcal{P}(2)) /\left(\theta^{-1}(3 R)\right) \cong \mathcal{P} e r m \otimes \mathcal{P}(\cong \mathcal{P} e r m \circ \mathcal{P})$.

Proof. We define a bijection $\xi: \mathcal{P} \operatorname{erm}(2) \otimes \mathcal{P}(2) \rightarrow(\mathcal{P}$ erm $\otimes \mathcal{P})(2)$ by

$$
\begin{aligned}
{\left[\alpha x_{1}, x_{2}\right] } & \mapsto e_{2}^{2} \otimes\left[x_{1}, x_{2}\right], \\
{\left[x_{1}, \alpha x_{2}\right] } & \mapsto e_{1}^{2} \otimes\left[x_{1}, x_{2}\right]
\end{aligned}
$$

Claim. This bijection is equivariant.

Hence it induces an operadic isomorphism $\xi: \mathcal{F}(\mathcal{P e r m}(2) \otimes \mathcal{P}(2)) \cong \mathcal{F}((\mathcal{P e r m} \otimes$ $\mathcal{P})(2))$. We show that this isomorphism induces the isomorphism of the proposition. It suffices to show that the image of $\theta^{-1}(3 R)$ by $\xi$ vanishes on $\mathcal{P} e r m \otimes \mathcal{P}$. The relation Ave vanishes in $\mathcal{P}$ erm $\otimes \mathcal{P}$, because

$$
\begin{array}{lll}
{\left[\alpha\left[\alpha x_{1}, x_{2}\right], x_{3}\right]^{\prime}} & \stackrel{\xi}{\mapsto} & e_{2}^{2} \otimes\left[x_{1}, x_{2}\right]^{\prime} \circ_{1} e_{2}^{2} \otimes\left[x_{1}, x_{2}\right] \stackrel{\pi}{\mapsto} e_{3}^{3} \otimes\left[\left[x_{1}, x_{2}\right], x_{3}\right]^{\prime}, \\
{\left[\alpha\left[x_{1}, \alpha x_{2}\right], x_{3}\right]^{\prime}} & \stackrel{\xi}{\mapsto} \quad e_{2}^{2} \otimes\left[x_{1}, x_{2}\right]^{\prime} \circ_{1} e_{1}^{2} \otimes\left[x_{1}, x_{2}\right] \stackrel{\pi}{\mapsto} e_{3}^{3} \otimes\left[\left[x_{1}, x_{2}\right], x_{3}\right]^{\prime},
\end{array}
$$

where $\pi$ is the projection onto $\mathcal{P} e r m \otimes \mathcal{P}$.

Claim. By a direct computation, one can show that

$$
\pi \circ \xi\left(\theta^{-1}\left(\left[\left[x_{a}, x_{b}\right], x_{c}\right]^{\prime} \overline{\mathrm{o}}_{j} \alpha \overline{\mathrm{o}}_{k} \alpha\right)\right)=e_{i}^{3} \otimes\left[\left[x_{a}, x_{b}\right], x_{c}\right]^{\prime},
$$

where $(a, b, c),(i, j, k) \in\{(1,2,3),(3,1,2),(2,3,1)\}$.

Therefore we obtain

$$
\pi \circ \xi: \theta^{-1}\left(R \bar{\circ}_{j} \alpha \bar{\circ}_{k} \alpha\right) \mapsto e_{i}^{3} \otimes R .
$$

The image $e_{i}^{3} \otimes R$ is zero on $\mathcal{P}$ erm $\otimes \mathcal{P}$, because $R$ is the relation of $\mathcal{P}$.

We will use this proposition in the next section. The relation $\theta^{-1}(3 R)$ is the basic properties of the derived brackets. Hence the operad $\mathcal{P} e r m \circ \mathcal{P}$ is completely determined by the properties that the derived brackets satisfy.

\subsection{An example}

We consider the derived brackets on dg Poisson algebras. Let $(P, \cdot,\{\cdot, \cdot\}, d)$ be a dg Poisson algebra. We assume that the degrees of the multiplications are both zero (or even). We denote by Poiss the operad of Poisson algebras. The derived bracket/product are defined by

$$
\begin{aligned}
& {[s x, s y]_{d}:=-(-1)^{|x|} s\{d x, y\},} \\
& s x *_{d} s y:=-(-1)^{|x|} s((d x) y) .
\end{aligned}
$$


From the symmetry of Poisson structures, we have $[1,2]^{d}=-[2,1]_{d}$ and $1 *^{d} 2=2 *_{d} 1$. Then $s P$ becomes a $\mathcal{P} e r m \circ \mathcal{P}$ oiss-algebra. The derived bracket is Leibniz (I) and the derived product $*_{d}$ is perm (II), and they are satisfying the three conditions below.

$$
\begin{aligned}
& {\left[s x, s y *_{d} s z\right]_{d}=[s x, s y]_{d} *_{d} s z+(-1)^{(|x|+1)(|y|+1)} s y *_{d}[s x, s z]_{d}} \\
& {\left[s x *_{d} s y, s z\right]_{d}=s x *_{d}[s y, s z]_{d}+(-1)^{(|x|+1)(|y|+1)} s y *_{d}[s x, s z]_{d}} \\
& {[s x, s y]_{d} *_{d} s z=-(-1)^{(|x|+1)(|y|+1)}[s y, s x]_{d} *_{d} s z .}
\end{aligned}
$$

Aguiar ([1]) introduced two new type of algebras, namely, prePoisson algebras and dual-prePoisson algebras. The latter is the Koszul dual of the former. A dualprePoisson algebra is defined to be an algebra equipped with two multiplications, [,] and $*$, satisfying (I), (II), (8), (9) and (10). Therefore the algebra of the derived bracket/product on a Poisson algebra is a dual-prePoisson algebra. From this observation, we obtain

Proposition 3.7. pre oiss $! \cong \mathcal{P} e r m \circ$ Poiss.

Proof. The relations (I), (II), (8), (9) and (10) are subrelations of $\theta^{-1}\left(3 R_{\mathcal{P}_{\text {oiss }}}\right)$, because they are the basic properties of the derived bracket/product. The dimension of the space of quadratic relations of prePoiss! is 30 :

$$
30=(6+9)+(6+6+3)
$$

where $(6+9)$ is the dimension of the space generated by (I) and (II), $(6+6+3)$ is the dimension of the space of generated by (8), (9) and (10). On the other hand, since $\operatorname{dim} R_{\mathcal{P}_{\text {oiss }}}=6, \operatorname{dim} \theta^{-1}\left(3 R_{\mathcal{P}_{\text {oiss }}}\right)=30$. The proof is completed.

Since $\mathcal{P}$ oiss $\cong \mathcal{P}$ oiss! and pre $\mathcal{L}$ ie $\cong \mathcal{P}$ erm! , we have prePoiss $\cong$ pre $\mathcal{L}$ ie $\bullet \mathcal{P}$ oiss.

\section{Koszul duality theory}

It was shown in $[5,6]$ Theorem 2.2.6 that the Koszul dual of the Manin white product $\mathcal{P}_{1} \circ \mathcal{P}_{2}$ is the Manin black product, $\mathcal{P}_{1}^{!} \bullet \mathcal{P}_{2}^{!}$(see also Appendix below). It is known that the Koszul dual of the operad Perm is the operad of preLie algebras, pre $\mathcal{L}$ ie (cf. [3, 4]). Hence we have

$$
(\mathcal{P} e r m \circ \mathcal{P})^{!}=\operatorname{pre} \mathcal{L} i e \bullet \mathcal{P}^{!},
$$

which implies that the operad pre $\mathcal{L} i e$ is closely related with the derived bracket construction. In this section, we discuss a Koszul duality for the derived bracket 
construction.

Let $\mathcal{P}$ be a binary quadratic operad and let $A$ be a $\mathcal{P}$-algebra equipped with multiplications $\left(*_{1}, \ldots, *_{n}\right)$. By definition, a Rota-Baxter operator (with weight zero) on $A$ is a linear endomorphism $\beta: A \rightarrow A$ satisfying the Rota-Baxter identity:

$$
\beta(x) *_{i} \beta(y)=\beta\left(\beta(x) *_{i} y+x *_{i} \beta(y)\right),
$$

for any $x, y \in A$ and for any $i$ (cf. [1] [2]). For example, the ordinary integral operator

$$
\beta(f)(x):=\int_{0}^{x} f(t) d t
$$

is a Rota-Baxter operator on the algebra of functions $C^{0}([0,1])$. In this sense, the Rota-Baxter operators are considered as a kind of dual-operators of differential operators. It is inferred that the integral/Rota-Baxter operators are the Koszul dual of the differential/averaging operators from some known observations (see [1] [2]). In Theorem 4.2 below, we give definite evidence that this statement is true.

Definition 4.1. Let $\mathcal{P}=\mathcal{P}(E, R)$ be an arbitrary binary quadratic operad and let $(A, \beta)$ be a $\mathcal{P}$-algebra with a Rota-Baxter operator. We call the following multiplications the dual-derived products.

$$
\beta(x) *_{i} y \text { and } x *_{i} \beta(y) .
$$

The original idea of the dual-derived products was given by Aguiar in [1].

Theorem 4.2. Let $\mathcal{P}=\mathcal{P}(E, R)$ be an arbitrary binary quadratic operad and let $\mathcal{P}^{!}\left(E^{\vee}, R^{\perp}\right)$ the Koszul dual and let $(A, \beta)$ a $\mathcal{P}^{!}$-algebra with a Rota-Baxter operator. The algebra of the dual-derived products is a pre $\mathcal{L}$ ie $\bullet \mathcal{P}^{!}$-algebra.

Proof. First we study the dual of Proposition 3.6. We recall (6) in Section 3.2. In the same way, we define 2-copies of $E^{\vee}$ :

$$
\operatorname{pre} \mathcal{L} i e(2) \otimes \mathcal{P}^{!}(2):=\left\langle\beta x_{1} * x_{2}, x_{1} * \beta x_{2} \mid x_{1} * x_{2} \in E^{\vee}\right\rangle,
$$

where $\beta$ is a label on copies (not Rota-Baxter operator). The $S_{2}$-module structure on $\operatorname{pre} \mathcal{L} i e(2) \otimes \mathcal{P}^{!}(2)$ is defined by the natural manner:

$$
\left(\beta x_{1} * x_{2}\right)(12)=\beta x_{2} * x_{1}=x_{1}{ }^{(12)} \beta x_{2},
$$

where $*^{(12)} \in E^{\vee}$ is the transposition of $*$. The operadic duality of $\operatorname{pre} \mathcal{L} i e(2) \otimes \mathcal{P}^{!}(2)$ and $\operatorname{Perm}(2) \otimes \mathcal{P}(2)$ is well-defined by

$$
\begin{aligned}
<\beta x_{1} * x_{2},\left[\alpha x_{1}, x_{2}\right]> & :=<x_{1} * x_{2},\left[x_{1}, x_{2}\right]> \\
<x_{1} * \beta x_{2},\left[x_{1}, \alpha x_{2}\right]> & :=<x_{1} * x_{2},\left[x_{1}, x_{2}\right]> \\
\text { otherwise } & :=0
\end{aligned}
$$


where the pairing of the right-hand side is the duality of $E^{\vee}$ and $E$. We set the 3-copies of $\mathcal{F}\left(E^{\vee}\right)(3)$, like $3 \mathcal{F}(E)(3)$,

$$
3 \mathcal{F}\left(E^{\vee}\right)(3):=\left(\mathcal{F}\left(E^{\vee}\right)(3) \overline{\mathrm{o}}_{2} \beta \overline{\mathrm{o}}_{3} \beta\right) \oplus\left(\mathcal{F}\left(E^{\vee}\right)(3) \overline{\mathrm{o}}_{3} \beta \overline{\mathrm{o}}_{1} \beta\right) \oplus\left(\mathcal{F}\left(E^{\vee}\right)(3) \overline{\mathrm{o}}_{1} \beta \overline{\mathrm{o}}_{2} \beta\right) .
$$

The duality of $3 \mathcal{F}(E)(3)$ and $3 \mathcal{F}\left(E^{\vee}\right)(3)$ is naturally defined. We recall the projection $\theta$ in Section 3.2. We consider the dual map of $\theta$ :

$$
\mathcal{F}\left(\operatorname{pre} \mathcal{L} i e(2) \otimes \mathcal{P}^{!}(2)\right)(3) \leftarrow 3 \mathcal{F}\left(E^{\vee}\right)(3): \theta^{\vee}
$$

The image of $3 R^{\perp}, \theta^{\vee}\left(3 R^{\perp}\right)$, is a quadratic relation in $\mathcal{F}\left(2 E^{\vee}\right)(3)$. As a corollary of Proposition 3.6, we obtain

Lemma 4.3. $\mathcal{F}\left(\operatorname{pre} \mathcal{L} i e(2) \otimes \mathcal{P}^{!}(2)\right) /\left(\theta^{\vee}\left(3 R^{\perp}\right)\right) \cong \operatorname{pre} \mathcal{L} i e \bullet \mathcal{P}^{!}$.

By a direct computation, we obtain

$$
\begin{aligned}
& \theta^{\vee}\left(\left(x_{i} * x_{j}\right) *^{\prime} x_{k} \overline{\mathrm{o}}_{j} \beta \overline{\mathrm{o}}_{k} \beta\right)=\left(x_{i} * \beta x_{j}\right) *^{\prime} \beta x_{k}, \\
& \theta^{\vee}\left(\left(x_{i} * x_{j}\right) *^{\prime} x_{k} \overline{\mathrm{o}}_{k} \beta \overline{\mathrm{o}}_{i} \beta\right)=\left(\beta x_{i} * x_{j}\right) *^{\prime} \beta x_{k},
\end{aligned}
$$

and from the condition, $<\theta^{\vee}(-)$, Ave $>=0$, we get the formal Rota-Baxter identity,

$$
\theta^{\vee}\left(\left(x_{i} * x_{j}\right) *^{\prime} x_{k} \bar{o}_{i} \beta \bar{o}_{j} \beta\right)=\beta\left(\beta x_{i} * x_{j}\right) *^{\prime} x_{k}+\beta\left(x_{i} * \beta x_{j}\right) *^{\prime} x_{k} .
$$

Now we prove the theorem. Let $r \in R^{\perp}$ be a quadratic relation of $\mathcal{P}^{\text {! }}$. Then one can write

$$
r=\sum_{\left(*, *^{\prime}\right)} C_{1}\left(x_{1} * x_{2}\right) *^{\prime} x_{3}+C_{2}\left(x_{3} * x_{1}\right) *^{\prime} x_{2}+C_{3}\left(x_{2} * x_{3}\right) *^{\prime} x_{1},
$$

where $C_{1}, C_{2}, C_{3}$ are structure constants. We obtain

$$
\begin{array}{r}
\theta^{\vee}\left(r \overline{\mathrm{o}}_{1} \beta \overline{\mathrm{o}}_{2} \beta\right)=\sum_{\left(*, *^{\prime}\right)} C_{1} \beta\left(\beta x_{1} * x_{2}\right) *^{\prime} x_{3}+C_{1} \beta\left(x_{1} * \beta x_{2}\right) *^{\prime} x_{3}+ \\
C_{2}\left(x_{3} * \beta x_{1}\right) *^{\prime} \beta x_{2}+C_{3}\left(\beta x_{2} * x_{3}\right) *^{\prime} \beta x_{1} .
\end{array}
$$

If $\beta$ is a real Rota-Baxter operator, that is, if the relation is represented on the algebra of the dual-derived products, then

$$
\begin{aligned}
\theta^{\vee}\left(r \overline{\mathrm{o}}_{1} \beta \overline{\mathrm{o}}_{2} \beta\right) & \stackrel{\text { rep }}{\rightarrow} \sum_{\left(*, *^{\prime}\right)} C_{1}\left(\beta x_{1} * \beta x_{2}\right) *^{\prime} x_{3}+C_{2}\left(x_{3} * \beta x_{1}\right) *^{\prime} \beta x_{2}+C_{3}\left(\beta x_{2} * x_{3}\right) *^{\prime} \beta x_{1} \\
& \left.=r \circ_{1} \beta \circ_{2} \beta=0 \quad \text { (on } A\right) .
\end{aligned}
$$

This holds for each $\theta^{\vee}\left(r \bar{\circ}_{i} \beta \bar{\circ}_{j} \beta\right),(i, j) \in\{(1,2),(2,3),(3,1)\}$. The proof of the theorem is completed. 
Since $\mathcal{L}$ ie $\bullet \mathcal{P} \cong \mathcal{P}$, we call pre $\mathcal{L}$ ie $\bullet \mathcal{P}$-algebras simply pre $\mathcal{P}$-algebras.

Corollary 4.4. ([12] Proposition 22). Let $A$ be a preP-algebra equipped with multiplications $\beta x_{1} *_{i} x_{2}$ and $x_{1} *_{i} \beta x_{2}$, where $i \in\{1, \ldots, \operatorname{dim} \mathcal{P}(2)\}$. We define a commutator associated with $*_{i}$,

$$
\left\{x_{1}, x_{2}\right\}_{i}:=\beta x_{1} *_{i} x_{2}+x_{1} *_{i} \beta x_{2} .
$$

The algebra of the commutators is a $\mathcal{P}$-algebra.

Proof. Let $r=r\left(*_{1}, *_{2}, \ldots\right)$ be a $\mathcal{P}$-algebra relation composed of $\mathcal{P}$-algebra multiplications. We have

$$
\sum_{(i, j) \in\{(1,2),(2,3),(3,1)\}} \theta^{\vee}\left(r \bar{o}_{i} \beta \bar{o}_{j} \beta\right)=r\left(\{\cdot, \cdot\}_{1},\{\cdot, \cdot\}_{2}, \ldots\right)=0
$$

The corollary above is considered to be a dual of Proposition 3.3, because Proposition 3.3 relies on the projection $\mathcal{P}$ erm $\rightarrow \mathcal{C}$ om and the above corollary relies on the dual map pre $\mathcal{L} i e \leftarrow \mathcal{L} i e$.

\section{Appendix}

A1. White products. Let $\mathcal{P}_{i}$ be a binary quadratic operad over $\left(E_{i}, R_{i}\right), i \in$ $\{1,2\}$.

Definition 5.1. (White products [5, 6] Section 2.2; [12] Section 3.2)

$$
\mathcal{P}_{1} \circ \mathcal{P}_{2}:=\frac{\mathcal{F}\left(E_{1} \otimes E_{2}\right)}{\Phi^{-1}\left(R_{1} \otimes \mathcal{F}\left(E_{2}\right)(3)+\mathcal{F}\left(E_{1}\right)(3) \otimes R_{2}\right)},
$$

where $\Phi$ is the unique operad morphism in the universal diagram below.

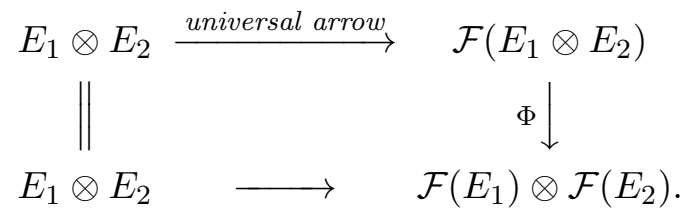

A2. Operadic dual spaces. Let $E$ be an $S_{n}$-module. The operadic dual of $E$, which is denoted by $E^{\vee}$, is a dual space of $E$ whose duality is defined by the pairing:

$$
<e, f>=\operatorname{sgn}(\sigma)<e \sigma, f \sigma>
$$

where $e \in E, f \in E^{\vee}$ and $\sigma \in S_{n}$. We consider the case of $n=2$. Let $e_{i}$ be a noncommutative multiplication and let $e_{i}^{(12)}$ the transposition of $e_{i}$ and let $e_{j}^{ \pm}$ 
be commutative $(+)$, anti-commutative $(-)$ multiplications, respectively. One can regard the duality as the pseudo-Euclidean metric defined by

$$
\begin{aligned}
<e_{i}, e_{j}> & =\delta_{i j}, \\
<e_{i}^{(12)}, e_{j}^{(12)}> & =-\delta_{i j}, \\
<e_{i}^{+}, e_{j}^{-}> & =\delta_{i j}, \\
<e_{i}^{-}, e_{j}^{+}> & =\delta_{i j},
\end{aligned}
$$

and all others 0 . The invariancy of the pairing above is built in the metric.

A3. Koszul dual. One can identify $\mathcal{F}(E)(3)$ with the 3 -copies $3(E \otimes E)$. This isomorphism is defined by

$$
e \circ_{1} e^{\prime}\left(x_{i}, x_{j}, x_{k}\right) \cong e \otimes e^{\prime} \otimes(i, j, k),
$$

where $(i, j, k) \in\{(1,2,3),(3,1,2),(2,3,1)\}$. For instance,

$$
\left[\left[x_{i}, x_{k}\right], x_{j}\right]^{\prime}=\left[\left[x_{k}, x_{i}\right]^{(12)}, x_{j}\right]^{\prime} \cong\left[x_{1}, x_{2}\right]^{\prime} \otimes\left[x_{1}, x_{2}\right]^{(12)} \otimes(k, i, j) .
$$

The duality of $E$ and $E^{\vee}$ is naturally extended to the duality of $\mathcal{F}(E)(3)$ and $\mathcal{F}\left(E^{\vee}\right)(3)$, via the isomorphism. More explicitly, the operadic duality of $\mathcal{F}(E)(3)$ and $\mathcal{F}\left(E^{\vee}\right)(3)$ is well-defined by

$$
<e \otimes e^{\prime} \otimes(i, j, k), f \otimes f^{\prime} \otimes(a, b, c)>:=<e, f><e^{\prime}, f^{\prime}>\delta_{i a} \delta_{j b} \delta_{k c} .
$$

This pairing is regarded as a metric.

Definition 5.2. Let $R$ be an $S_{3}$-subspace of $\mathcal{F}(E)(3)$, i.e., quadratic relation and let $R^{\perp} \subset \mathcal{F}\left(E^{\vee}\right)(3)$ the orthogonal space of $R$ with respect to the operadic duality. The Koszul dual of $\mathcal{P}:=\mathcal{F}(E) /(R)$ is defined to be the operad

$$
\mathcal{P}^{!}:=\mathcal{F}\left(E^{\vee}\right) /\left(R^{\perp}\right) .
$$

Since $R^{\perp \perp} \cong R$, we have $\mathcal{P}^{! !} \cong \mathcal{P}$.

A4. Black products. We consider the dual map of $\Phi$ on the third component.

$$
\mathcal{F}\left(E_{1}^{\vee} \otimes E_{2}^{\vee}\right)(3) \leftarrow \mathcal{F}\left(E_{1}^{\vee}\right)(3) \otimes \mathcal{F}\left(E_{2}^{\vee}\right)(3): \Psi,
$$

where $\Psi=\Phi(3)^{\vee}$.

Definition 5.3. (Black product [5, 6] Section 2.2; [12] Section 4.3)

$$
\mathcal{P}_{1}^{!} \bullet \mathcal{P}_{2}^{!}:=\frac{\mathcal{F}\left(E_{1}^{\vee} \otimes E_{2}^{\vee}\right)}{\Psi\left(R_{1}^{\perp} \otimes \mathcal{F}\left(E_{2}^{\vee}\right)(3) \cap \mathcal{F}\left(E_{1}^{\vee}\right)(3) \otimes R_{2}^{\perp}\right)} .
$$

Since $\mathcal{P} ! ! \cong \mathcal{P}$, the black product is defined for any binary quadratic operads. It is obvious that $\left(\mathcal{P}_{1} \circ \mathcal{P}_{2}\right)^{!} \cong \mathcal{P}_{1}^{!} \bullet \mathcal{P}_{2}^{!}$, because $\Psi\left(R_{1}^{\perp} \otimes \mathcal{F}\left(E_{2}^{\vee}\right)(3) \cap \mathcal{F}\left(E_{1}^{\vee}\right)(3) \otimes R_{2}^{\perp}\right)$ is the orthogonal space of $\Phi^{-1}\left(R_{1} \otimes \mathcal{F}\left(E_{2}\right)(3)+\mathcal{F}\left(E_{1}\right)(3) \otimes R_{2}\right)$. 


\section{References}

[1] M. Aguiar. Pre-Poisson algebras. Lett. Math. Phys. 54 (2000), no. 4, 263-277.

[2] M. Aguiar and J-L Loday. Quadri-algebras. J. Pure Appl. Algebra 191 (2004), no. $3,205-221$.

[3] F. Chapoton. Un endofoncteur de la categorie des operades. Lecture Notes in Mathematics, 1763. Springer-Verlag, Berlin (2001), 105-110.

[4] F. Chapoton and M. Livernet. Pre-Lie algebras and the rooted trees operad. Internat. Math. Res. Notices (2001), no. 8, 395-408.

[5] V. Ginzburg and M. Kapranov. Koszul duality for operads. Duke Math. J. 76 (1994), no. 1, 203-272.

[6] V. Ginzburg and M. Kapranov. Erratum to: "Koszul duality for operads" Duke Math. J. 80 (1995), no. 1, 293.

[7] Y. Kosmann-Schwarzbach. From Poisson algebras to Gerstenhaber algebras. Ann. Inst. Fourier (Grenoble) 46 (1996), no. 5, 1243-1274.

[8] Y. Kosmann-Schwarzbach. Derived brackets. Lett. Math. Phys. 69 (2004), 6187.

[9] J-L. Loday and T. Pirashvili. Universal enveloping algebras of Leibniz algebras and (co)homology. Math. Ann. 296 (1993), no. 1, 139-158.

[10] J-L. Loday. Dialgebras. Lecture Notes in Mathematics, 1763. Springer-Verlag, Berlin, (2001), 7-66.

[11] M. Markl, S. Shnider and J. Stasheff. Operads in algebra, topology and physics. Mathematical Surveys and Monographs, 96. American Mathematical Society, Providence, RI, (2002). $\mathrm{x}+349$ pp.

[12] B. Vallette. Manin products, Koszul duality, Loday algebras and Deligne conjecture. J. Reine Angew. Math. 620 (2008), 105-164.

Science University of Tokyo.

Wakamiya 26 Shinjuku Tokyo Japan.

e-mail: K_Uchino[at]oct.rikadai.jp 